\title{
Promoter hypermethylation of PTPL1, PTPN6, DAPK, p16 and 5-azacitidine inhibits growth in DLBCL
}

\author{
WENMING WANG ${ }^{1}$, JING WANG ${ }^{1}$, ZHENGQIAN LI ${ }^{2}$, MINGXIA ZHU $^{1}$, \\ $\mathrm{ZHE} \mathrm{ZHANG}^{3}$, YANFANG WANG ${ }^{1}$ and $\mathrm{HONGMEI} \mathrm{JING}^{1}$ \\ Departments of ${ }^{1}$ Hematology, ${ }^{2}$ Anesthesiology and ${ }^{3}$ Urology, Peking University Third Hospital, Beijing 100191, P.R. China
}

Received July 3, 2015; Accepted August 18, 2015

DOI: $10.3892 /$ or.2015.4347

\begin{abstract}
Aberrant hypermethylation of $\mathrm{CpG}$ islands of tumor suppressor is one of the mechanisms for epigenetic loss of gene function. In the present study, the methylation status of the promoter regions of protein tyrosine phosphatase (PTPN) 6 , DAPK, and p16 were studied using methylation-specific polymerase chain reaction (MSP) in 26 diffuse large $\mathrm{B}$ cell lymphoma (DLBCL) lymphomas. In OCI-LY1 cell line, gene methylation status, expression of PTPL1 and its reactivation by DNA demethylation was determined by PCR and on the protein level by western blotting. ELISA-like reaction was used to detect global DNA methylation measurement. Induction of apoptosis by 5-azacitidine was analyzed by Annexin V/ PI staining and flow cytometry. Our results show that hypermethylation of the PTPN6 gene promoter region was found in $15.4 \%(4 / 26)$, the DAPK gene promoter region in $30.8 \%(8 / 26)$, the p16 gene promoter region in $7.7 \%$ (2/26). Notably, we identified that PTPL1 was hypermethylated and transcriptionally silenced in OCI-LY1 cell line. The expression of PTPL1 was re-inducible by 5 -azacytidine. 5-azacytidine also inhibits the proliferation and decreases the global methylation level of the OCI-LY1 cell line. We can conclude from our study that a higher prevalence of methylation of PTPL1, PTPN6, DAPK and p16 occur in DLBCL. Our data also highlights 5-azacytidine as a potential therapeutic candidate for DLBCL. Further studies are required to substantiate the role of methylation of PTPL1, PTPN6, DAPK and p16 as a marker in diffuse large B cell lymphoma.
\end{abstract}

\section{Introduction}

Lymphomas are solid tumors of the immune system. Diffuse large B cell lymphoma (DLBCL) is the most common lymphoid neoplasm (1). The most common symptom of

Correspondence to: Professor Hongmei Jing, Department of Hematology, Peking University Third Hospital, 49 North Garden Road, Haidian, Beijing 100191, P.R. China

E-mail: hongmeijing@medmail.com.cn

Key words: protein tyrosine phosphatase 1, PTPN6, DAPK, p16, diffuse large B cell lymphoma, methylation, 5-azacitidine
DLBCL is lymphadenopathy. General symptoms may include loss of weight, decrease in performance status, fever and night sweats (2). Although the majority of patients with DLBCL can be cured with standard rituximab, cyclophosphamide, doxorubicin, vincristine, and prednisone (R-CHOP), patients who fail R-CHOP have dismal outcomes (3). DLBCLs inherit the cytosine methylation patterns instability that exist in the germinal center B cells from which DLBCLs arise and display variable degrees of epigenetic heterogeneity. Greater epigenetic heterogeneity is linked with poor clinical outcome (4). Many novel classes of drugs are in development that may improve the treatment of DLBCL, either as single agent or in combination (5). It is clear, however, that epigenetic alterations provide an additional series of targets that can be pharmacologically modified and offer great potential to improve patient outcomes (6).

In recent years, it has become apparent that aberrant DNA hypermethylation of tumor suppressor genes can result in their inappropriate transcriptional silencing, which contributes to loss of checkpoints and related functions in cancer (7). 5-aza-2-deoxycitidine and 5-azacitidine are applied to the treatment of MDS that incorporate into DNA and inactivate DNMT by forming a covalent bond between the 5-azacytosine ring and the enzyme (8). DNA methyltransferases (DNMTs), including DNMT1, DNMT3A, and DNMT3B, can methylate DNA specifically at cytosines were found to be overexpressed in 48,13 and $45 \%$ of de novo DLBCLs, respectively, and correlate with advanced clinical stage (9).

Protein tyrosine phosphatase (PTPL1), is also known as PTPN13, FAP-1, PTP-BAS, PTP1E, is a large non-transmembrane PTP at $270 \mathrm{kDa}(10)$. Hypermethylation of the PTPL1 gene promoter is involved in a variety of cancers, such as non-small cell lung cancer, esophageal cancer, gastric and hepatocellular tumors $(11,12)$. Hypermethylation of PTPN6, DAPK, p16 has been reported in many human tumors (13-15).

In the present study we applied MSP to determine the methylation status of the PTPN6, DAPK, p16 gene in 26 DLBCL. To evaluate the effects of 5-azacytidine, we studied cell proliferation, and apoptosis, as well as DNA methylation status in association with mRNA and protein expression levels of PTPL1 in the DLBCL cell line OCI-LY1. The results obtained from this study contributes to a better understanding of the role of methylation of tumor suppressor 
Table I. Sequences of primers used for PCR analysis.

\begin{tabular}{|c|c|c|c|}
\hline Gene & Forward & Reverse & Temperature $\left({ }^{\circ} \mathrm{C}\right)$ \\
\hline \multicolumn{4}{|l|}{ MS-PCR } \\
\hline PTPL1-M & CGAGTAGTTTTAGCGGTTAC & AAAACCTTCTAACGCGAACGA & 58 \\
\hline PTPN6-M & TGTAGTTTTCGGAGTTAGTGTCGCGC & CCTACGATCGAAAACGACGCGAACG & 59 \\
\hline DAPK-M & GGATAGTCGGATCGAGTTAACGTC & CCCTCCCAAACGCCGA & 60 \\
\hline p16-M & TTATTAGAGGGTGGGGCGGATCGC & GACCCCGAACCGCGACCGTAA & 60 \\
\hline \multicolumn{4}{|l|}{ RT-PCR } \\
\hline PTPL1 & GCGCTCCAGTAGCAGGAC & TCATCTGTAAATGACACACTAC & 55 \\
\hline BCL2 & СCTCTGTTTGATTTCTCCTGGCTGT & TGGAAAGCGAATCTATGTTTACAGG & 56 \\
\hline BAX & AACATGGAGCTGCAGAGGATG & CTGGTCTTGGATCCAGCCAGCCAACAG & 56 \\
\hline hCNT1 & CCTCACCTGTGTGGTCCTCA & AGACCССTCTTAAACCAGAGC & 57 \\
\hline hENT1 & TCTCCAACTCTCAGCCCACCAA & CCTGCGATGCTGGACTTGACCT & 58 \\
\hline hENT2 & ACCATGCCCTCCACCTACAG & GGGCCTGGGATGATTTTATTG & 55 \\
\hline$\beta$-actin & CCTTGCACATGCCGGAG & GCACAGAGCCTCGCCTT & 60 \\
\hline
\end{tabular}

gene in DLBCL and epigenetic pathways represent relevant and promising therapeutic targets in DLBCL, highlighting that 5 -azacytidine may offer a potential new therapeutic approach to improve the poor outcomes associated with DLBCL.

\section{Materials and methods}

Cell line. The OCI-LY1 cell line, derived from DLBCL, was maintained in IMDM medium (Gibco-Invitrogen, Carlsbad, CA, USA) supplemented with $10 \%$ fetal bovine serum (Hyclone, Logan, UT, USA), and $1 \%$ antibiotics (Gibco-Invitrogen). Cells were incubated at $37^{\circ} \mathrm{C}$ in a humid atmosphere at $5 \% \mathrm{CO}_{2}$ and split every 2-3 days depending on cell density.

Patients. The formalin-fixed paraffin-embedded (FFPE) tissues of 26 patients with de novo DLBCL with available tumor specimen at diagnosis were included in this study. The archived FFPE tissues were obtained from the Department of Pathology, Peking University Third Hospital. The histological diagnoses were established according to World Health Organization classifications. All participants were well informed, and provided a written consent form.

Bisulfite modification. For the methylation analysis, genomic DNA was extracted from cell lines and clinical samples using the E.Z.N.A ${ }^{\circledR}$ Tissue DNA kit (Omega Bio-Tek, Lilburn, GA, USA). DNA (200 ng) was processed for bisulfite modification using a BisulFlash DNA Modification kit (Epigentek, New York, NY, USA) according to the manufacturer's instructions. The bisulfite-modified DNA was stored at $-20^{\circ} \mathrm{C}$ or used immediately.

Methylation-specific PCR. DNA methylation patterns were determined by methylation-specific PCR (MSP) after bisulfite treatment of DNA. Bisulfite-modified DNA was amplified using the previously described primers PTPL1-M, PTPN6-M, DAPK-M, p16-M (Table I). Each MSP reaction was repeated to confirm the results.
$R T-P C R$. Briefly, total RNA was isolated from cultured cells with TRIzol reagent (Invitrogen) and concentrations assessed using a NanoDrop spectrophotometer (Thermo Fisher Scientific, Waltham, MA, USA). Then, $1 \mu \mathrm{g}$ of total RNA was reverse-transcribed with the Goscript ${ }^{\mathrm{TM}}$ Reverse Transcription system (Thermo Fisher Scientific). The primer sequences used are shown in Table I.

Global DNA methylation measurement. Genome-wide methylation pattern was measured by the MethylFlash kit (Epigentek, Farmingdale, NY, USA) following the manufacturer's instructions. Cells were seeded in a 6-well plate at the density of $2.5 \times 10^{5} / \mathrm{ml}$, and then treated with $1,5,10,20 \mu \mathrm{M}$ 5 -azacytidine for 3 days for an additional $24 \mathrm{~h}$. DNA (total $100 \mathrm{ng}$ ) is detected using capture and detection antibodies and then quantified through an ELISA-like reaction by reading the absorbance in a microplate spectrophotometer at $450 \mathrm{~nm}$. All investigations were performed in triplicates using 2 independent replicates.

Western blotting. OCI-LY1 cells were seeded at the density of $2.5 \times 10^{5} / \mathrm{ml}$ and treated with $1,5,10,20 \mu \mathrm{M} 5$-azacytidine at 0 , 24 and $48 \mathrm{~h}$, a total of three times. Total protein was isolated from cultured cells and quantified using a BCA protein assay kit. Samples ( $60 \mu \mathrm{g}$ protein/lane) were separated by 8 and $15 \%$ sodium dodecyl sulfate-polyacrylamide gel electrophoresis (SDS-PAGE). After transfer to a nitrocellulose membrane, proteins were incubated with the following primary antibodies overnight at $4^{\circ} \mathrm{C}$ : anti-PTPL1 (1:200); anti-caspase-3 (1:500), and anti- $\beta$-actin $(1: 1,000)$ (all from Santa Cruz Biotechnology, Inc., Santa Cruz, CA, USA). Fluorescently labeled secondary antibodies (1:10,000; LI-COR Biosciences, Lincoln, NE, USA) were used to detect the binding of the primary antibodies. The bound proteins were visualized by scanning the membranes in an Odyssey Infrared Imaging system (LI-COR Biosciences) (16).

Cell viability assay. Cellular viability was assessed with CCK-8 assay as described previously. In brief, OCI-LY1 cells 
A
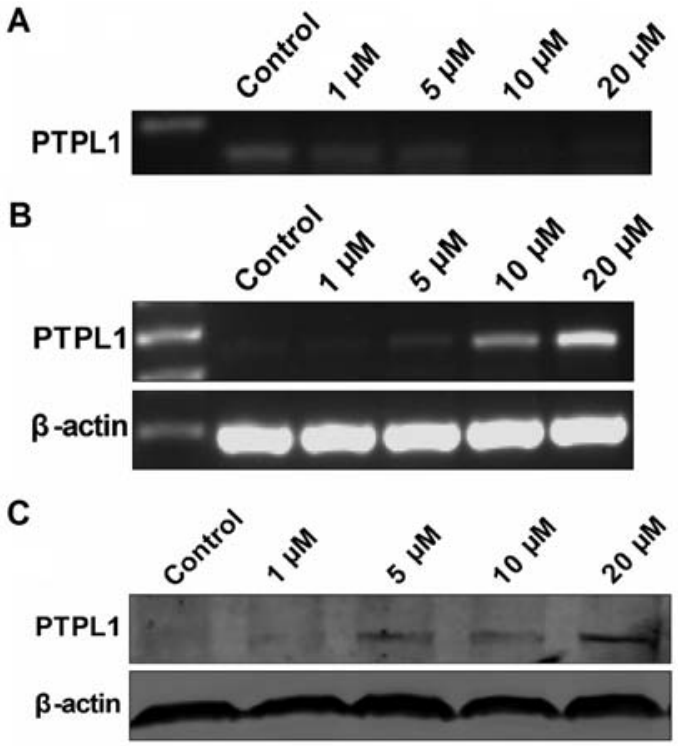

Figure 1. PTPL1 gene expression and methylation in OCI-LY1 cell line. (A) PTPL1 is methylated in OCI-LY1 cell line and can be demethylated by 5-azacytidine. (B and C) RT-PCR and western blotting showing the restored PTPL1 expression in OCI-LY1 cell lines.

were plated in 96-well plates at $\sim 25,000$ cells/well with $100 \mu \mathrm{l}$ culture medium. The cells were randomly divided into the control group and the test groups medially with three duplicates per group. They were then treated with 5 -azacytidine at $0.1,0.5,1,2,5,10,20$ and $50 \mu \mathrm{M}$ for $12,24,48$, and $72 \mathrm{~h}$, respectively. Ten microliters of CCK-8 (Dojindo, Kumamoto, Japan) accompanied every sample in each well. Non-treated cells were considered as control and incubated in similar conditions for the same period of time. After $2 \mathrm{~h}$ incubation at $37^{\circ} \mathrm{C}$ in the dark, the absorption value $\mathrm{A}$ of each well was detected at the wavelength of $450 \mathrm{~nm}$ in a Quant spectrophotometer. All procedure was repeated three times and average values were calculated. Cell inhibition rate (I\%) was calculated using the following equation: $\mathrm{I} \%=[\mathrm{A}($ control $)-$ A(treated)]/[A(control)-A(blank)] x 100\%.

Mitochondrial membrane potential. OCI-LY1 cells were treated with 5-azacytidine at $0.1,0.5,1,2,5,10,20$ and $50 \mu \mathrm{M}$ for 24,48 , and $72 \mathrm{~h}$, respectively. The collapse of mitochondrial transmembrane potential $(\Delta \psi \mathrm{m})$ was assessed with the probe JC-1, a sensitive fluorescent dye. Cells were analyzed with FACSCanto (BD Biosciences, San Jose, CA, USA). In healthy cells, where mitochondrial potentials remain depolarized, JC-1 forms complexes of J-aggregates showing punctate red fluorescence at $590 \mathrm{~nm}$ emission wavelength, in apoptotic cells, JC-1 remains in the monomeric form showing diffused green fluorescence at $530 \mathrm{~nm}$ emission wavelength.

Flow cytometric analysis for apoptosis. To further quantify the percentage of cells undergoing apoptosis, we performed double staining with Annexin V-FITC and propidium iodide (PI) staining. Briefly, $2.5 \times 10^{5}$ cells $/ \mathrm{ml}$ were plated in 6 -well plates as described above treated with $1,5,10$, and $20 \mu \mathrm{M} 5$-azacytidine for 3 days for an additional $24 \mathrm{~h}$. After treatment, the cells were stained
Table II. Results of DNA methylation.

\begin{tabular}{lrr}
\hline Gene & Methylation & Demethylation \\
\hline PTPN6 & $15.4 \%(4 / 26)$ & $84.6 \%(22 / 26)$ \\
DAPK & $30.8 \%(8 / 26)$ & $69.2 \%(18 / 26)$ \\
p16 & $7.7 \%(2 / 26)$ & $92.3 \%(24 / 26)$ \\
\hline
\end{tabular}

with Annexin V-FITC/PI according to the manufacturer's instructions. The apoptotic cells were analyzed by flow cytometry (BD Biosciences).

Immunohistochemistry. For immunohistochemical analysis, paraffin sections were deparaffinized in xylene for $15 \mathrm{~min}$ three times and rehydrated through decreasing concentrations of ethanol (100, 95, and 80\% 5 min, 2 times each). Endogenous peroxidase activity was blocked by incubation of the slides in $3 \% \mathrm{H}_{2} \mathrm{O}_{2}$ /phosphate-buffered saline, and non-specific binding sites were blocked with goat serum. Antigens were retrieval by microwave irradiation for $3 \mathrm{~min}$ in $\mathrm{pH} 6.0$ citric buffer and thawed for 15 min. Primary antibody was anti-PTPL1 (1:50; Santa Cruz Biotechnology, Inc.) and the detection of bound antibodies was performed using HRP polymer kit, employing diaminobenzidine (DAB) as a chromogen (17).

Statistical analysis. The data are expressed as mean \pm SEM and analyzed using SPSS 21.0 for Windows (SPSS, Inc., Chicago, IL, USA). For comparisons of multiple groups, one-way ANOVA tests followed by LSD multiple comparison tests was performed. Statistical significance was set at $\mathrm{P}<0.05$.

\section{Results}

PTPL1 gene promoter methylation pattern and 5-azacytidine treatment for demethylation and re-expression of PTPL1 in OCI-LY1 cell line. Methylation of the PTPL1 gene was found in OCI-LY1 cell line (Fig. 1A). To experimentally assess whether PTPL1 promoter hypermethylation affects PTPL1 expression, we first examined the PTPL1 levels in OCI-LY1 cell line (Fig. 1B and C). To test whether PTPL1 expression is epigenetically regulated via promoter methylation in lymphoma, we treated cell lines with 5-azacytidine, leading to DNA demethylation. OCI-LY1 cell line with hypermethylated promoters failed to express OCI-LY1, except when subjected to prior treatment with 5-azacytidine.

Treatment with 5-azacytidine was able to restore PTPL1 expression in OCI-LY1 cell line at both mRNA and protein levels (Fig. 1B and C). Dosage of 5-azacytidine (10 and $20 \mu \mathrm{M})$ invariably produced a relatively clear response.

To further clarify the relationship between PTPL1 promoter methylation and expression, the methylation status of PTPL1 after 5-azacytidine treatment was observed. Treated with 5-azacytidine was able to reduce PTPL1 promoter methylation in the OCI-LY1 cell line (Fig. 1A). The results confirmed that hypermethylation of PTPL1 was responsible for gene silencing since DNA demethylation resulted in reactivation of PTPL1 transcription in OCI-LY1 cell lines. 


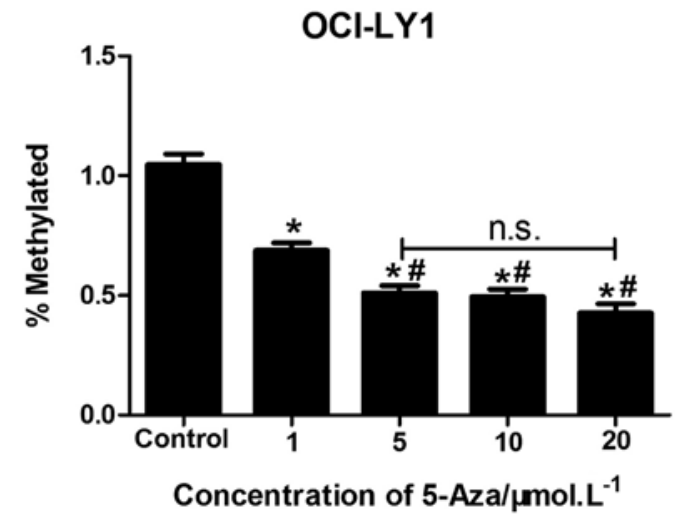

Figure 2. Genome-wide methylation pattern changes after 5-azacytidine treatment. Compared with the control cells, treatment of OCI-LY1 cell line with $1,5,10$, and $20 \mu \mathrm{M} 5$-azacytidine led to a significantly decreased methylation level for both concentrations. ${ }^{*} \mathrm{P}<0.05$ vs. control group; ${ }^{~} \mathrm{P}<0.05$ vs. $1 \mu \mathrm{M}$ group.

PTPN6, DAPK and pl6 gene promoter hypermethylation by $M S-P C R$ analysis. We examined the DNA from 26 formalin fixed paraffin-embedded (FFPE) tissues of patients with DLBCL (Table II). Hypermethylation of the PTPN6 gene promoter region was found in $15.4 \%$ (4/26), the DAPK gene promoter region in $30.8 \%(8 / 26)$, the p16 gene promoter region in $7.7 \%(2 / 26)$.

Genome-wide methylation pattern changes after 5-azacytidine treatment. A low global methylation status was observed in all groups. Compared with the control cells, treatment of OCI-LY1 cell line with 1, 5, 10, and $20 \mu \mathrm{M} 5$-azacytidine led to a significantly decreased methylation level for both concentrations (Fig. 2). $\mathrm{P}<0.05$ vs. control group; $\mathrm{P}<0.05$ vs. $1 \mu \mathrm{M}$ group.

5-azacytidine inhibits OCI-LY1 cell line proliferation. To determine whether 5-azacytidine affects the growth of OCI-LY1 cells, we measured the proliferation of OCI-LY1 cells treated with 5-azacytidine. To confirm the effects of 5-azacytidine on OCI-LY1 cell proliferation, the CCK-8 assay was used to assess cell proliferation and quantify cell viability. Fig. 3 shows that 5-azacytidine markedly inhibited the growth of OCI-LY1 cells in a dose- and time-dependent manner.

5-azacytidine decreases mitochondrial membrane potential, translocates Baxand Bcl2, and increases apoptosis in OCI-LY1 cell line. Loss of mitochondrial membrane potential is a sign of the apoptotic process in cells. The mitochondria-mediated apoptosis pathway plays a central role in apoptosis induced by the caspase-dependent pathway. 5-azacytidine treatment of OCI-LY1 resulted in a dosage-and time-dependent increase in the percentage of green fluorescent positive cells (Fig. 4A).

Members of the $\mathrm{Bcl} 2$ family play a critical role through their pro-apoptotic ( $\mathrm{Bax})$ or anti-apoptotic $(\mathrm{Bcl} 2)$ activities. Results shows that the expression of Bax mRNA and $\mathrm{Bcl} 2$ mRNA was decreased concentration-dependently at 24, 48 , and $72 \mathrm{~h}$, but on the whole, $\mathrm{Bax} / \mathrm{Bcl} 2$ ratio in favor of apoptosis was increased (Fig. 4B).
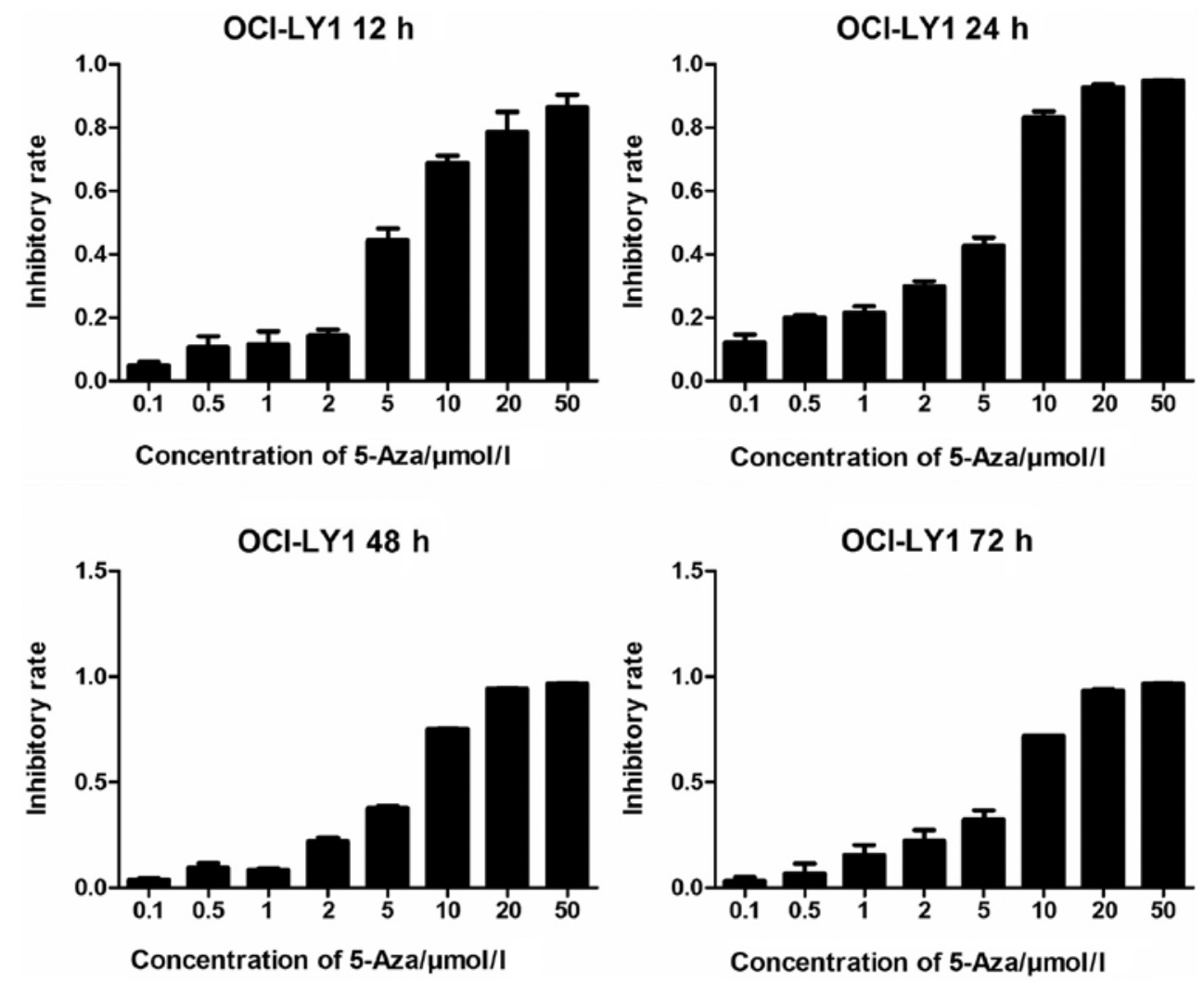

Figure 3. 5-azacytidine inhibits growth of the OCI-LY1 cell line. Cell proliferation was assayed using the Cell Counting Kit-8 (CCK-8). OCI-LY1 cells were seeded at a density of $2.5 \times 10^{5} / \mathrm{ml}$ and incubated in triplicate in the presence of various concentrations of 5 -azacytidine for $12,24,48$, and $72 \mathrm{~h}$, respectively. 
A
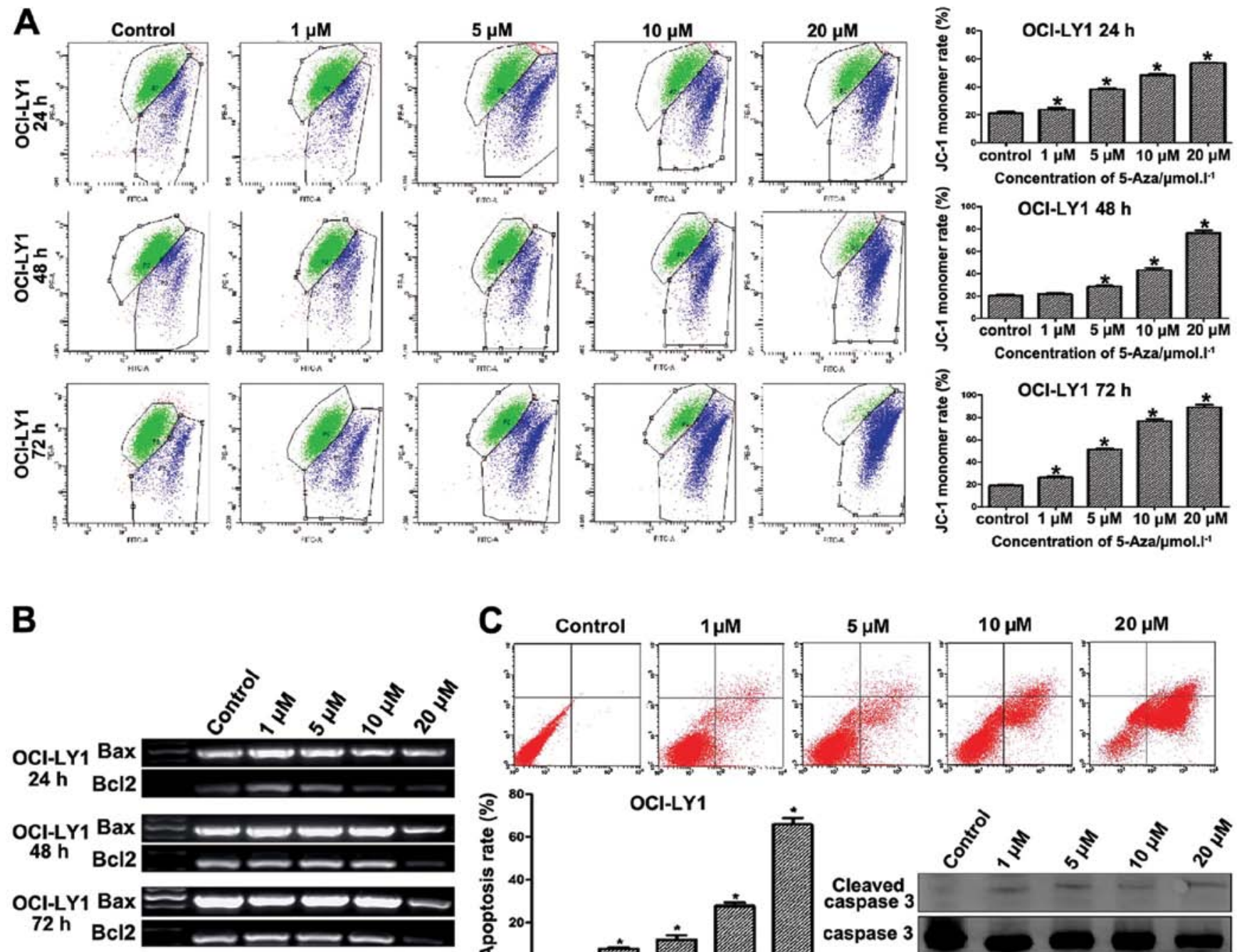

$20 \mu \mathrm{M}$
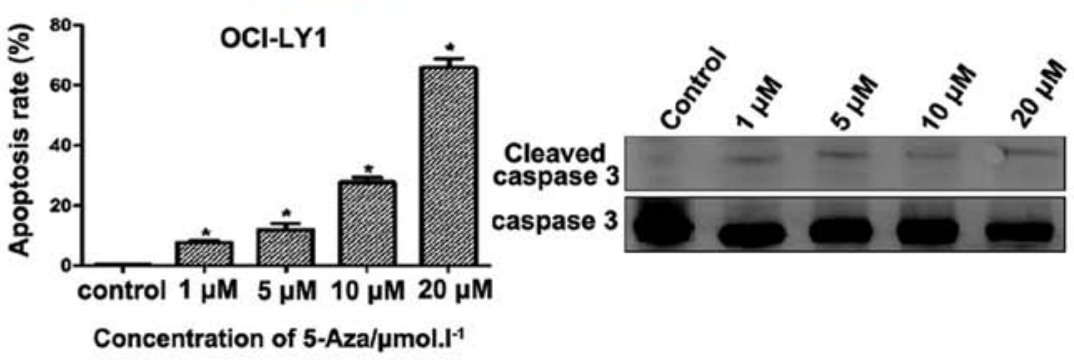

Figure 4. 5-azacytidine suppresses growth and promotes apoptosis of U266 and H929 cell lines. (A) 5-azacytidine reduces mitochondrial membrane potential (B) 5-azacytidine upregulates the Bax/Bcl2 ratio. (C) 5-azacytidine-mediated apoptosis. ${ }^{*} \mathrm{P}<0.05$.

We next determined whether 5-azacytidine-mediated decrease of OCI-LY1 cell viability was the result of apoptosis. With the increasing dose of 5-azacytidine, for OCI-LY1 the apoptosis rate considerable increase of early (Annexin $\left.\mathrm{V}^{+} / \mathrm{PI}^{-}\right)$ apoptotic cells at the concentration of $1 \mu \mathrm{M}, 5 \mu \mathrm{M}, 10 \mu \mathrm{M}$, $20 \mu \mathrm{M}$ of 5-azacytidine, respectively. Caspases are important regulators of apoptosis. It is well known that the activation of caspase-3 during apoptosis causes the cleavage of PARP, a major indicator enzyme of apoptosis. We also measured the expression of caspase- 3 protein. After $72 \mathrm{~h}$ for an additional $24 \mathrm{~h}$ of treatment with 5 -azacytidine $(1 \mu \mathrm{M}, 5 \mu \mathrm{M}, 10 \mu \mathrm{M}$, $20 \mu \mathrm{M})$, the levels of cleavage caspase-3 in OCI-LY1 cells were increased (Fig. 4C).

Immunohistochemical study. The expression pattern of PTPL1 in DLBCL tissue was analyzed by immunohistochemistry using a specific antibody reacting against PTPL1 protein. Three DLBCLs in our series were PTPL1-negative. The remaining 7 cases were PTPL1-positive (Fig. 5).
Expression of hCNT1, hENT1 and hENT2 after 5-azacytidine treatment. RT-PCR was used to observed the expression level of hNT, to clarify their role in antitumor efficacy of 5-azacytidine (Fig. 6). Compared with control, for hCNT1, the expression was decreased after treatment with 5-azacytidine in OCI-LY1 cell lines; for hENT1 and hENT2, the changes of expression were complicated, there was a slight increase before the decrease.

\section{Discussion}

Advances in molecular genetics have vastly improved our understanding of the molecular pathways that may drive oncogenesis. Both genetic and epigenetic phenomena interact in tumorigenesis (18). DNA methyltransferase and histone methyltransferase inhibitors are emerging as viable therapeutic approaches to erase aberrant epigenetic programming, suppressing DLBCL growth, and overcome chemotherapy resistance (19,20). 5-azacytidine shows antitumor effect 

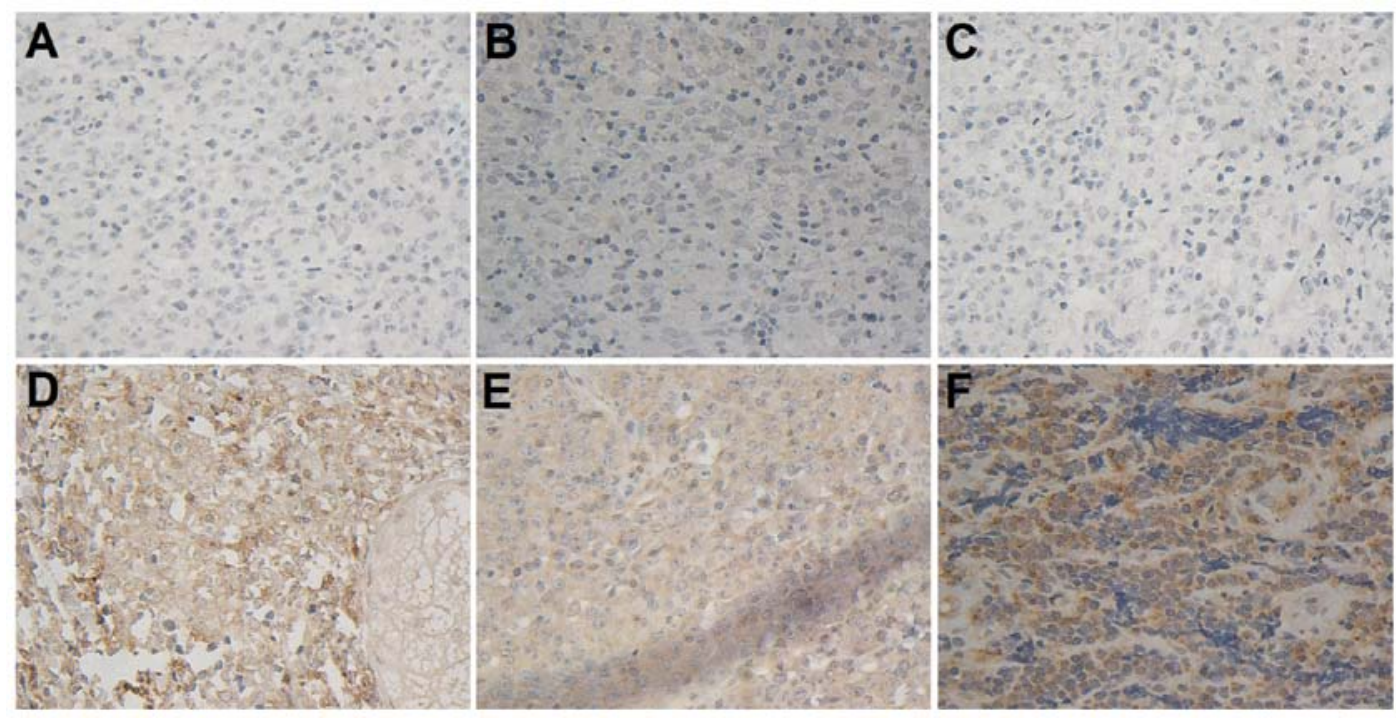

Figure 5. Immunohistochemical staining of DLBCL. Three DLBCLs (A-C) in our series were PTPL1-negative. The remaining 7 cases (D-F as representative) were PTPL1-positive.

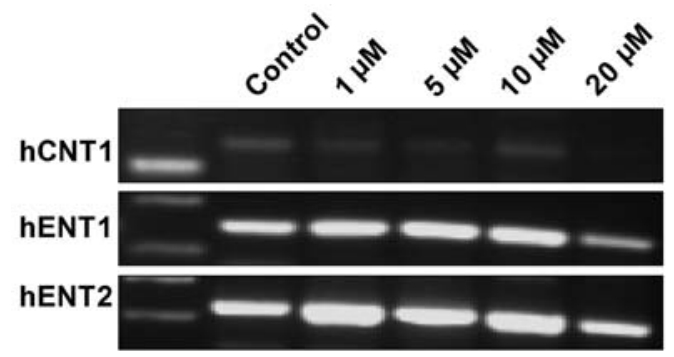

Figure 6. The expression level of hCNT1, hENT1 and hENT2 after 5-azacytidine treatment. RNA was isolated from OCI-LY1 cell line treated with 1,5 , 10 , and $20 \mu \mathrm{M}$ 5-azacytidine for 3 days for an additional $24 \mathrm{~h}$. Expression of hCNT1, hENT1 and hENT2 was evaluated by RT-PCR.

through two major mechanisms, suppressing methylation and developing cytotoxicity by inhibiting protein synthesis. The proportion of each mechanism mainly depends on concentration, its cytotoxic activity increases with concentration; whereas, DNMT inhibition peaks at low concentrations and abates at higher concentrations. Although there have been numerous studies on the antitumor effects of 5-azacytidine, its action on DLBCL is still obscure. Our experiments explored the effects of 5-azacytidine on cell proliferation, apoptosis, the alterations of gene-specific methylation status and global methylation status, to clarify the relationship between 5-azacytidine and DNA methylation, and suggested new potential clinical uses as an epigenetic therapy in DLBCL.

The loss of tumor suppressor gene (TSG) function can lead to a set of functional capabilities for malignant growth, usually including self-sufficiency in growth signals, insensitivity to growth-inhibitory signals, evasion of apoptosis, limitless replicative potential, sustained angiogenesis, and tissue invasion and metastasis (21). TSG may become epigenetically silenced by hypermethylation of $\mathrm{CpG}$ islands located in their promoter regions and epigenetic silencing of TSG is potentially reversible (22). In our study, the frequency of methylation of the PTPN6, DAPK, p16 gene in DLBCL was demonstrated. DAPK is involved in the regulation of apoptosis, p16 is a well-known tumor suppressor gene that plays a key role in cell cycle control, PTPN6 usually functions as a negative regulator in signal transduction (23). In a study of 148 DLBCL patients, DAPK methylation was observed in $89 \%$ and the methylation of p16 is linked with poor clinical outcome (24). In addition, DAPK methylation is associated with poor overall- and disease-specific survival in a large cohort of DLBCL patients uniformly treated with rituximab. One previous study showed an incidence of promoter hypermethylation of $23 \%$ each for p16 and p15 in oral squamous cell carcinoma. The methylation of the PTPN6 gene promoter was detected in many B-cell leukemia/lymphoma cell lines as well as in patient specimens, including diffuse large B-cell lymphoma (methylation frequency, 93\%), MALT lymphoma (82\%), mantle cell lymphoma (75\%), plasmacytoma (100\%) and follicular lymphoma (96\%), and also found in high-grade glioma, childhood acute lymphoblastic leukemia, and multiple myeloma (25). In our previous study, the promoter of gene PTPL1 was methylated in 59.6\% (28/47). We show here that hypermethylation of the PTPN6 gene promoter region was found in $15.4 \%(4 / 26)$, the DAPK gene promoter region was found in $30.8 \%(8 / 26)$, the p16 gene promoter region was found in $7.7 \%(2 / 26)$. On the whole, our results are consistent with the previous results, but the methylation rate is lower than in the previous study. We assume different type of specimens is the reason. Our specimens were taken from formalin-fixed paraffin-embedded (FFPE) tissues and some of them are kept for many years.

Thus, the global DNA methylation level was decreased after treatment with 5-azacytidine for three days. For 5-azacytidine, DNMT inhibition decreases with concentration and abates at higher concentrations. Compared with control group, all groups were decreased significantly, compared with $1 \mu \mathrm{M}$ group, 5, 10, and $20 \mu \mathrm{M}$ groups were decreased significantly, but there is no statistical difference among the 5,10 , and $20 \mu \mathrm{M}$ groups. The demethylation of genomic DNA can promote the differentiation of DLBCL cells, but in our study, this was not 
detected. Further experiments are necessary to show whether the changes in differentiation status of DLBCL cells were due to 5-azacytidine concentration. Our experiments indicated that 5-azacytidine inhibited the proliferation of OCI-LY1 cells for $12,24,48$, and $72 \mathrm{~h}$, its biological effects on cell growth varied depending on concentration and time of exposure.

In our present study, PTPL1 was methylated in OCI-LY1 cells and was silenced in these two cell lines at mRNA and protein levels. After treatment with 5-azacytidine for three days, all cell lines show PTPL1 mRNA and protein re-expression and reduced methylation level of the PTPL1 promoter. In a previous study, using a total of 82 tumor cell lines, showed that the expression of PTPL1 was frequently downregulated or silenced in non-Hodgkin's lymphoma (94\%), Hodgkin's lymphoma (50\%), breast (30\%), gastric (60\%) and hepatocellular $(67 \%)$ carcinoma cell lines. In summary, PTPL1 may be a putative tumor suppressive gene in DLBCL. In our study, PTPL1 expression was downregulated in the 30\% DLBCL cases. The result supports that the downregulation of PTPL1 may have a relation with the occurrence and development of DLBCL.

The present study aimed to explore the molecular mechanisms underlying the induction of cytotoxic effects by 5 -azacytidine in OCI-LY1 cells. Following 5-azacytidine treatment, dose-dependent increases in the apoptotic rate were observed. The BCL2 family members play a central role in regulating programmed cell death and arbitrating the cellular fate through an accurate balance between pro-apoptotic (BAX, $\mathrm{BAK}$, and $\mathrm{BH} 3$-only proteins) and pro-survival (BCL2 and its closest homologues, BCLXL, BCLW and MCL-1) factors. The ratio of $\mathrm{Bax} / \mathrm{Bcl} 2$ could ultimately tip the balance toward cell survival or cell death (26). Reverse transcription-polymerase chain reaction analysis demonstrated that 5-azacytidine treatment upregulated the ratio of Bax/Bcl-2 and flow cytometry showed loss of mitochondrial membrane potential. The decreased mitochondrial membrane potential involved the release of cytochrome $c$ and Smac/DIABLO, leading to activation of the caspase cascade, and ultimately apoptosis. Caspase-3 is a member of the caspase family of enzymes, which are the major inducers of apoptosis and is often measured in the context of research into antitumor drugs that target apoptosis $(27,28)$. Further studies were performed to examine the effect of 5-azacytidine on cell apoptosis and the activity of caspase-3. The induction of cell death by 5 -azacytidine mainly occurs via programmed cell death, as indicated by Annexin V-fluorescein isothiocyanate and PI staining. Cells treated with 1, 5, 10, and $20 \mu \mathrm{M} 5$-azacytidine for 3 days detected by FACS demonstrated 5-azacytidine induced apoptosis in a dose-dependent manner. In addition our results showed that the activity of caspase-3 after treated with 5-azacytidine for 3 days increased significantly. This increase of caspase- 3 was concomitant with the apoptosis of cell lines. Accordingly, the decrease in Bcl-2, increase in Bax and, finally, the activation of caspase- 3 in OCI-LY1 cells indicated that the apoptotic process induced by 5-azacytidine was irreversible.

Nucleoside transporters play important physiological roles by regulating intra- and extra-cellular concentrations of purine and pyrimidine (deoxy) nucleosides, including inflammation, cardiovascular function and nutrient transport across the blood-brain barrier (29). In addition, nucleoside transport plays its role in pathological conditions such as cardiovascular disease and cancer. Focusing on nucleoside analogs as antineoplastic agents, it may be a potential therapeutic strategy to manipulate the activity of nucleoside transporters. In our study, for hENT1 and hENT2, the change of expression was complicated, there was a slight increase before the decrease; for hCNT1, the expression was decreased after treatment with 5-azacytidine in OCI-LY1 cell line. Moreover, hENT1 expression in leukemia cell lines appears to be a key determinant of 5-azacytidine triggered cytotoxicity. Nucleoside transporters are responsible for the cellular uptake of currently used anticancer nucleoside-derived drugs, thus these membrane proteins might play a significant role in nucleoside-based chemotherapy.

Drug resistance is a major problem for patients treated with small molecular reagents such as 5-azacytidine and decitabine, but the mechanisms are poorly understood (30). The final decrease of hCNT1, hENT1 and hENT2 after treatment with 5-azacytidine may suggest that decreasing levels of these transporters is a possible mechanism for acquiring resistance to DNMT inhibitors. We believe our study will give some clues for the development of novel chemical entities to measure nucleoside transport activity at the cellular and organismal level.

Taken together, our observations demonstrated that 5 -azacytidine has potent effects on growth inhibition, induction of caspase-dependent apoptosis in the OCI-LY1 cell line. Epigenetic dysregulation is involved in PTPN6, DAPK, p16. The loss of PTPL1 mRNA is the consequence of PTPL1 methylation and can be reversed by 5 -azacytidine. The study highlights the value of 5-azacytidine as epigenetic potential therapeutic candidate for DLBCL.

\section{References}

1. Campo E, Swerdlow SH,Harris NL, Pileri S, Stein H and Jaffe ES: The 2008 WHO classification of lymphoid neoplasms and beyond: Evolving concepts and practical applications. Blood 117: 5019-5032, 2011.

2. Young RM, Shaffer AL III, Phelan JD and Staudt LM: B-cell receptor signaling in diffuse large B-cell lymphoma. Semin Hematol 52: 77-85, 2015.

3. Sehn LH, Berry B, Chhanabhai M, Fitzgerald C, Gill K, Hoskins P, Klasa R, Savage KJ, Shenkier T, Sutherland J, et al: The revised International Prognostic Index (R-IPI) is a better predictor of outcome than the standard IPI for patients with diffuse large B-cell lymphoma treated with R-CHOP. Blood 109: 1857-1861, 2007.

4. Jiang Y and Melnick A: The epigenetic basis of diffuse large B-cell lymphoma. Semin Hematol 52: 86-96, 2015.

5. Clozel T, Yang S, Elstrom RL, Tam W, Martin P, Kormaksson M, Banerjee S, Vasanthakumar A, Culjkovic B, Scott DW, et al: Mechanism-based epigenetic chemosensitization therapy of diffuse large B-cell lymphoma. Cancer Discov 3: 1002-1019, 2013.

6. Shaknovich R, Geng H, Johnson NA, Tsikitas L, Cerchietti L, Greally JM, Gascoyne RD, Elemento O and Melnick A: DNA methylation signatures define molecular subtypes of diffuse large B-cell lymphoma. Blood 116: e81-e89, 2010.

7. Robaina MC, Mazzoccoli L, Arruda VO, Reis FR, Apa AG, de Rezende LM and Klumb CE: Deregulation of DNMT1, DNMT3B and miR-29s in Burkitt lymphoma suggests novel contribution for disease pathogenesis. Exp Mol Pathol 98: 200-207, 2015.

8. Zhao X, Yang F, Li S, Liu M, Ying S, Jia X and Wang X: CpG island methylator phenotype of myelodysplastic syndrome identified through genome-wide profiling of DNA methylation and gene expression. Br J Haematol 165: 649-658, 2014. 
9. Amara K, Ziadi S, Hachana M, Soltani N, Korbi S and Trimeche M: DNA methyltransferase DNMT3b protein overexpression as a prognostic factor in patients with diffuse large B-cell lymphomas. Cancer Sci 101: 1722-1730, 2010.

10. Freiss G and Chalbos D: PTPN13/PTPL1: An important regulator of tumor aggressiveness. Anticancer Agents Med Chem 11: 78-88, 2011.

11. Castilla C, Chinchón D, Medina R, Torrubia FJ, Japón MA and Sáez C: PTPL1 and PKC contribute to proapoptotic signalling in prostate cancer cells. Cell Death Dis 4: e576, 2013.

12. Glondu-Lassis M, Dromard M, Chavey C, Puech C, Fajas L, Hendriks W and Freiss G: Downregulation of protein tyrosine phosphatase PTP-BL represses adipogenesis. Int J Biochem Cell Biol 41: 2173-2180, 2009.

13. Bodoor K, Haddad Y, Alkhateeb A, Al-Abbadi A, Dowairi M, Magableh A, Bsoul N and Ghabkari A: DNA hypermethylation of cell cycle (p15 and p16) and apoptotic (p14, p53, DAPK and TMS1) genes in peripheral blood of leukemia patients. Asian Pac J Cancer Prev 15: 75-84, 2014.

14. Don KR, Ramani P, Ramshankar V, Sherlin HJ, Premkumar P and Natesan A: Promoter hypermethylation patterns of P16, DAPK and MGMT in oral squamous cell carcinoma: A systematic review and meta-analysis. Indian J Dent Res 25 : 797-805, 2014

15. Kupčinskaitė-Noreikienè R, Skiecevičienė J, Jonaitis L, Ugenskienė R, Kupčinskas J, Markelis R, Baltrènas V, Sakavičius L, Semakina I, Grižas S, et al: $\mathrm{CpG}$ island methylation of the MLH1, MGMT, DAPK, and CASP8 genes in cancerous and adjacent noncancerous stomach tissues. Medicina (Kaunas) 49: 361-366, 2013.

16. Zhang Z, Nie F, Kang C, Chen B, Qin Z, Ma J, Ma Y and Zhao X Increased periostin expression affects the proliferation, collagen synthesis, migration and invasion of keloid fibroblasts under hypoxic conditions. Int J Mol Med 34: 253-261, 2014.

17. Liang Y, Li Z, Mo N, Li M, Zhuang Z, Wang J, Wang Y and Guo $\mathrm{X}$ : Isoflurane preconditioning ameliorates renal ischemia-reperfusion injury through antiinflammatory and antiapoptotic actions in rats. Biol Pharm Bull 37: 1599-1605, 2014.

18. Saleem M, Abbas K, Manan M, Ijaz H, Ahmed B, Ali M, Hanif M, Farooqi AA and Qadir MI: Review - epigenetic therapy for cancer. Pak J Pharm Sci 28: 1023-1032, 2015.

19. Pike BL, Greiner TC, Wang X, Weisenburger DD, Hsu YH, Renaud G, Wolfsberg TG, Kim M, Weisenberger DJ, Siegmund KD, et al: DNA methylation profiles in diffuse large B-cell lymphoma and their relationship to gene expression status. Leukemia 22: 1035-1043, 2008.
20. Gibaja V, Shen F, Harari J, Korn J, Ruddy D, Saenz-Vash V, Zhai H, Rejtar T, Paris CG, Yu Z, et al: Development of secondary mutations in wild-type and mutant EZH2 alleles cooperates to confer resistance to EZH2 inhibitors. Oncogene: Apr 20, 2015 (Epub ahead of print). http://dx.doi.org/10.1038/onc.2015.114.

21. Wang S and Wang Z: Epigenetic aberrant methylation of tumor suppressor genes in small cell lung cancer. $\mathbf{J}$ Thorac Dis 5: 532-537, 2013.

22. Mehta A, Dobersch S, Romero-Olmedo AJ and Barreto G: Epigenetics in lung cancer diagnosis and therapy. Cancer Metastasis Rev 34: 229-241, 2015

23. Witzig TE, Hu G, Offer SM, Wellik LE, Han JJ, Stenson MJ, Dogan A, Diasio RB and Gupta M: Epigenetic mechanisms of protein tyrosine phosphatase 6 suppression in diffuse large B-cell lymphoma: Implications for epigenetic therapy. Leukemia 28: 147-154, 2014.

24. Kristensen LS, Treppendahl MB, Asmar F, Girkov MS, Nielsen HM, Kjeldsen TE, Ralfkiaer E, Hansen LL and Grønbæk K: Investigation of MGMT and DAPK1 methylation patterns in diffuse large B-cell lymphoma using allelic MSP-pyrosequencing. Sci Rep 3: 2789, 2013.

25. Koyama M, Oka T, Ouchida M, Nakatani Y, Nishiuchi R, Yoshino T, Hayashi K, Akagi T and Seino Y: Activated proliferation of B-cell lymphomas/leukemias with the SHP1 gene silencing by aberrant $\mathrm{CpG}$ methylation. Lab Invest 83: 1849-1858, 2003.

26. Scarfò L and Ghia P: Reprogramming cell death: BCL2 family inhibition in hematological malignancies. Immunol Lett 155: 36-39, 2013

27. Gupta S and Knowlton AA: HSP60, Bax, apoptosis and the heart. J Cell Mol Med 9: 51-58, 2005.

28. Zeng J, Chen S, Li N, Chen L, Su J, Niu G, Zhu S and Liang Y: Sasanquasaponin from Camellia oleifera Abel. induces apoptosis via Bcl-2, Bax and caspase-3 activation in HepG2 cells. Mol Med Rep 12: 1997-2002, 2015.

29. Chang C, Swaan PW, Ngo LY, Lum PY, Patil SD and Unadkat JD: Molecular requirements of the human nucleoside transporters hCNT1, hCNT2, and hENT1. Mol Pharmacol 65: 558-570, 2004.

30. Pastor-Anglada M and Pérez-Torras S: Nucleoside transporter proteins as biomarkers of drug responsiveness and drug targets. Front Pharmacol 6: 13, 2015. 